\title{
Kommunale Rolle bei der Umsetzung des SGB II stärken
}

Plädoyer für kommenale Zuständigkeiten

\author{
Tina Hofmann und Thomas Niermann
}

Die Arbeitsvermittlung nach dem SGB II muss aus verfassungsrechtlichen Gründen neu geregelt werden. Diese Chance sollte der Gesetzgeber nutzen, die Verantwortung von Städten und Landkreisen zu stärken und alle in einer Kommune tätigen Akteure der Sozial- und Wirtschaftspolitik einzubinden.

Nach dem Urteil des Bundesverfassungsgerichts vom Dezember 2007, mit dem die Zusammenarbeit von Arbeitsagenturen und Kommunen in den Arbeitsgemeinschaften (ARGEN) für verfassungswidrig erklärt wurden, hatten der Staatssekretär im Bundesarbeitsministerium, Detlef Scheele, und der Leiter der Bundesagentur für Arbeit, Frank-Jürgen Weise, sofort eine »Lösung " parat: Bei der Bundesagentur für Arbeit sollten eigene Geschäftseinheiten zur Umsetzung des SGB II - sogenannte kooperative Jobcenter - eingerichtet werden. Das $\mathrm{Zu}$ sammenwirken von Arbeitsagentur und Kommune wäre auf der Grundlage von freiwilligen Vereinbarungen zu organisieren.

Diese Vorschläge haben einen Entrüstungssturm ausgelöst; beim Arbeitsministerium hagelte es kritische Stellungnahmen aus Verbänden, ARGEN und Kommunen. Kernpunkt der Kritik: Das Modell fußt auf einer getrennten Aufgabenwahrnehmung von Kommunen und Arbeitsagenturen mit der Folge, dass Geld- und Sachleistungen auseinanderfallen. Freiwillige Vereinbarungen seien viel zu vage, um eine einheitliche Hilfeleistung sicherzustellen.

Der Paritätische Wohlfahrtsverband

Tina Hofmann ist Referentin für Jugendsozialarbeit im Paritätischen Wohlfahrtsverband -

Gesamtverband e. V.

E-Mail jugendsozialarbeit@paritaet.org Thomas Niermann ist Abteilungsleiter Soziale Arbeit und internationale Kooperation im Paritätischen Wohlfahrtsverband - Gesamtverband e. V. E-Mail alsoz@paritaet.org halten bleiben, weil die Leistungen zur Eingliederung und zur Sicherung des Lebensunterhalts wie auch die psychosozialen Hilfen nicht von unterschiedlichen Leistungsträgern erbracht werden. Unschlagbare Vorteile haben die Kommunen auch mit den Möglichkeiten, die Arbeitsmarktpolitik mit weiteren kommunalen Gestaltungsfeldern strategisch zu verknüpfen. Dies gilt genauso für die Jugendhilfe, Bildungsarbeit und Schulentwicklung wie für die regionale Wirtschaftsförderung und Infrastrukturentwicklung.

Nach den vorläufigen Ergebnissen der bundesweiten Wirkungsforschung zum Systemvergleich zwischen ARGEN und Optionskommunen (1) nutzen die Kommunen lokale Handlungsspielräume, um die Arbeitsmarktpolitik mit der regionalen Wirtschaftsförderung zu verknüpfen und daraus Synergieeffekte zu erzielen. Die Kommunen haben mehr Kompetenz und Erfahrung in der Betreuung und Förderung von arbeitsmarktfernen Personengruppen wie sozial benachteiligte Jugendliche, Wohnungslose oder Suchtkranke. Für diese Personengruppen müssen sozialintegrative Hilfen an den Schnittstellen anderer Leistungsgesetze (z. B. SGB VIII, XII) erschlossen werden, die in kommunaler Umsetzungsverantwortung liegen. Offenbar sind Langzeitarbeitslose und schwer vermittelbare Personengruppe mehr im Fokus der Kommunen als der heutigen ARGEN, so erste Ergebnisse der Wirkungsforschung. Die Kommunen legen in der Ausrichtung ihrer Geschäftspolitiken und Fördermaßnahmen mehr Wert auf Angebote zur sozialen Absicherung und Stabilisierung der Langzeitarbeitslosen. Sie arbeiten außerdem häufiger mit der kommunalen Kinder- und Jugendhilfe sowie freien Trägern der Jugendhilfe zusammen, als dies bei den ARGEN der Fall ist. Insofern wird den Optionskommunen mit den vorläufigen Ergebnissen der 
bundesweiten Wirkungsforschung ein gutes Zwischenzeugnis ausgestellt.

Nach den Erfahrungen des Paritätischen Wohlfahrtsverbandes gibt es ein klares Plus für die Optionskommunen auch bei den lokalen Handlungsspielräumen. Während die ARGEN die lokale Ausgestaltung ihrer Arbeitsmarktpolitik durch zentrale Vorgaben der Bundesagentur für Arbeit immer wieder stark eingeschränkt sehen, nutzen die Kommunen ihre Möglichkeiten, um lokal angepasste und zielgruppengenaue Förderangebote zu entwickeln. Die überregionale Arbeitsvermittlung, ein Aufgabengebiet, in dem die Vorteile bei der Arbeitsagentur liegen, spielt nur in einigen Regionen, vor allen Dingen in Ostdeutschland, eine wesentliche Rolle. Dies sind alles wesentliche Gründe für kommunale Zuständigkeiten nach dem SGB II.

Nach den Vorschlägen des Bundesarbeitsministers sollen die ARGEN zu "Zentren für Arbeit und Grundsicherung « weiterentwickelt werden. Bund und Kommunen würden per Bundesgesetz verpflichtet, ihre Aufgaben nach dem SGB II in diesen neuen Zentren wahrzunehmen. Wie auch die ARGEN könnten die neuen Zentren einheitlich Geldleistungen auszahlen und Bescheide erlassen. Sie würden aber über kein eigenes Personal verfügen, sondern auf die Mitarbeiterinnen der Kommunen und der Bundesagentur für Arbeit zurückgreifen müssen. Entscheidendes Gremium zur Kooperation der beiden Behörden soll die Trägerversammlung sein, in der insbesondere das örtliche Arbeitsmarktprogramm abgestimmt werden soll. Gemeinsam getroffene Entscheidungen könnten aber jederzeit von einem der beiden Partner zurükkgenommen werden; jeder Träger behält ein »Letztentscheidungsrecht « über seinen Aufgabenbereich. Außerdem ist vorgesehen, dass der Bund die Rechts- und Fachaufsicht über die Trägerversammlungen führt.

\section{Kompromissmodell ist einer Optionslösung nicht überlegen}

Dass die neuen Zentren für Arbeit und Grundscherung den Optionskommunen überlegen sein werden, ist zweifelhaft. Die einheitliche Annahme von Anträgen und Leistungsbescheiden wie auch die geplante gemeinsame Leistungserbringung stellt sicherlich einen wesentlichen Fortschritt gegenüber dem Modell des kooperativen Jobcenters, nicht aber gegenüber dem heutigen Optionsmodell dar.

Wie auch den ARGEN wird den Zentren für Arbeit und Grundsicherung die Auswahl und Entwicklung eines qualifizierten Mitarbeiterstamms durch fehlende Personalkompetenzen erheblich erschwert. Ein abgestimmtes Handeln droht durch das »Letztentscheidungsrecht « der beiden Behörden unterlaufen zu werden.

Damit ist die kritische Anfrage begründet, ob in Nachfolge der ARGEN lokal handlungsfähige und leistungsstarke Organisationseinheiten entstehen werden. Die Bundesländer, deren Zustimmung eine neue SGB II-Struktur erforderlich ist, akzeptieren die Vorschläge jedenfalls noch nicht. die Förderarbeit kommt vielerorts $\mathrm{zu}$ kurz. Längerfristig aufgebaute zielgerichtete Förderungen gerade für benachteiligte Personengruppen müssen besser entwickelt und die Zusammenarbeit von freien Trägern und Kommunen in der Förderpraxis verbessert werden. (2)

Die Planung und Umsetzung der lokalen Arbeitsmarktpolitik ist auch als gemeinsame Gestaltungsaufgabe der Optionskommunen, der lokalen Wirtschaft und den Beteiligten des örtlichen Arbeitsmarkts (insbesondere freie Wohlfahrtspflege, Vertretungen der Arbeitgeber und Arbeitnehmer) zu verstehen. Dafür sollten sich die Kommunen stärker als bisher um die vom Gesetzgeber vorgesehene örtliche Zusammenarbeit der Beteiligten bemühen. Ob ihnen im Vergleich zu den ARGEN ein größerer Gestaltungsspielraum in der lokalen Arbeitsmarktpolitik

\section{»Die Politik muss die Handlungs- optionen für die örtliche Praxis erweitern"}

\section{Perspektiven und Handlungs- bedarfe für Optionskommunen}

Entgegen der ursprünglichen Planungen, die Zukunft der Optionskommunen (auch) von den Ergebnissen der Wirkungsforschung zum Systemvergleich zwischen ARGEN und Optionskommunen abhängig zu machen, gibt es demnach wohl grundlegende politische Weichenstellungen, bevor der Endbericht der Forscher vorliegt und bewertet werden kann. In Zukunft wird es aller Voraussicht nach auch keinen vom Gesetzgeber initiierten Leistungswettbewerb der Modelle mehr geben. Das gibt Anlass, auch für die Optionskommunen Handlungsbedarfe und Ansätze für ihre Weiterentwicklung zu benennen.

Zentrale Herausforderung für die Optionskommunen ist eine bessere individuelle Förderung. Vielen Optionskommunen fehlen qualifizierte und längerfristig beschäftigte Mitarbeiterinnen und Mitarbeiter. Die Verpflichtung, Eingliederungsvereinbarungen abzuschließen, wird häufig nur formal erfüllt. Die fachliche und inhaltliche Unterstützung für erhalten bleibt, hängt von der bevorstehenden Gesetzgebung zur Instrumentenreform ab. Geht es nach den Plänen des Bundesarbeitsministeriums, werden die Fördermöglichkeiten so stark auf das Regelinstrumentarium des SGB III begrenzt, dass eine gute Förderung gerade für arbeitsmarktferne Personen massiv behindert ist. Es bleibt zu hoffen, dass die Politik den Willen aufbringt, die Handlungsoptionen für die örtliche Praxis zu erweitern. Dafür müssen den Grundsicherungsträgern die Möglichkeiten gegeben werden, eigens entwickelte Förderleistungen umzusetzen.

Was aber, wenn Optionskommunen einen flexibilisierten Instrumentenkoffer missbrauchen würden, um sich von eigenen kommunalen Aufgaben zu entlasten und sich am Eingliederungstitel des Bundes schadlos zu halten? Derartige Bedenken, die beispielsweise im Umfeld des Bundesarbeitsministeriums geäußert werden, sollten durchaus erwogen werden, nachdem Kommunen mit der Einführung des SGB II ihre Ausgaben in der Jugendsozialarbeit zurückgefahren haben. Vieles spricht dafür, für bestimmte 
Förderleistungen wie niedrigschwellige Hilfen an der Schnittstelle zur Jugendoder Sozialhilfe anteilige Mitfinanzierungen anderer Leistungsträger, beispielsweise des Jugend- bzw. Sozialamts, vorzusehen. Ein Beispiel hierfür ist die Regelung $\mathrm{zu}$ den Aktivierungshilfen für Jugendliche.

In der Debatte um eine Weiterentwicklung des SGB II wird von kommunaler Seite häufig zu Recht gefordert, der »sozialintegrative Auftrag « des SGB II müsse gestärkt werden. Die Förderung im SGB II sollte also nicht alleine auf eine schnelle Vermittlung und Überwindung der Hilfebedürftigkeit gerichtet werden, sondern längerfristige und niedrigschwellige Hilfen sollten auch arbeitsmarktferne Personen unterstützen. Den Handlungsbedarf zeigt nicht zuletzt der SGB IIJahresbericht 2007 auf. (3) Darin dokumentiert die Bundesagentur für Arbeit Defizite in der Förderung von Langzeitarbeitslosen. Eine stärkere sozialintegrative Ausrichtung des SGB II bedarf eines stärkeren finanziellen Engagements der Kommunen, weil die psychosozialen Hilfen wie beispielsweise Schuldner- und Suchtberatungsangebote bei einem verstärkten Hilfeansatz mehr zum Einsatz kommen und die Leistungen ergänzender kommunaler Hilfesysteme wie der Sozial- und Jugendhilfe einbezogen werden müssten.

Im Verhältnis zwischen den Trägern der Grundsicherung und freien Trägern sollten die Vorgaben des $\$ 17$ SGB II stärker Beachtung finden. Dieser sieht vor, auf die vorhandenen Leistungen der freien Träger zurückzugreifen. Dass freie Träger vielerorts hochwertige und lokal passgenaue Hilfen für Arbeitslose bereitstellen, ist nur ein Grund, diese Regelung in der Praxis stärker zu nutzen. Es ergäbe sich weiterhin die Möglichkeit für Kommunen, ihre eigene Auftraggeberrolle stärker von der Leistungserbringung zu trennen, um Doppelrollen und damit einhergehende Interessengegensätze zu vermeiden. Der Deutsche Landkreistag empfiehlt in einer aktuellen Publikation zur Umsetzung der sozialen Leistungen nach SGB II die »Abgrenzung von Aufgaben und Zuständigkeiten für eine optimale Ausgestaltung von Leistungsprozessen «. (4) In der Publikation wird weiter ausgeführt, die Kommunen stünden als Auftraggeber in der Verantwortung, die strategische Steuerung sozialer Leistungen zu gewährleisten. Ausgespart wird bei diesen Ausführungen der häufige Praxisfall, dass die Kommunen sowohl Auftraggeber sozialer Aufgaben als auch Leistungserbringer sind. Aus der Doppelrolle heraus

\section{Was sind eigentlich Optionskommen?}

Das Optionsmodell ist ein Feldversuch für die Betreuung von Arbeitslosen in Deutschland. In 69 Kommunen sind für die Bezieher des ab Anfang 2005 bestehenden Arbeitslosengeldes II nicht die Bundesagentur für Arbeit und die Kommunen gemeinsam zuständig, sondern ausschließlich Städte oder Landkreise. Diese werden auch als Optionskommunen bezeichnet. Das Modell ist Ergebnis eines Kompromisses zwischen der rot-grünen Bundesregierung und der CDU/CSU-Opposition aus dem Jahr 2004. Die Opposition hatte ursprünglich eine ausschließliche Zuständigkeit der Kommunen in ganz Deutschland gefordert. Sie erhoffte sich dadurch mehr Nähe zum Bürger und höheren Konkurrenzkampf zwischen den Kommunen um den besten Erfolg und neue Modelle der Vermittlung von Langzeitarbeitslosen. Als Kompromiss mit dem Regierungsmodell der Arbeitsgemeinschaft wurde vereinbart, dass 69 Kommunen (Anzahl der Mitglieder des Bundesrats) im Rahmen des Optionsmodells die Gewährung des Arbeitslosengeldes II in eigener Zuständigkeit bearbeiten, um die Vor- und Nachteile dieses Modells in der Praxis zu testen und mit den anderen Varianten der Zuständigkeit (Arbeitsgemeinschaft, getrennte Trägerschaft) zu vergleichen. Das Optionsmodell wird nun seit 1. Januar 2005 befristet auf sechs Jahre (bis 31. Dezember 2010) ausprobiert. Neu für die teilnehmenden Städte und Gemeinden ist, dass sie sich nun auch um die Vermittlung von Arbeitslosen kümmern. Bisher waren hierfür die örtlichen Agenturen für Arbeit zuständig. Für das von den Kommunen ausgezahlte Arbeitslosengeld II und die Verwaltungskosten kommt der Bund auf.

Quelle: Internet http://de.wikipedia.org

kann es schwierig werden, eine klare und konsequente Steuerung gegenüber den Leistungserbringern in der eigenen Organisationseinheit wahrzunehmen und allen Leistungserbringern gleichermaßen (fair) gegenüberzutreten.

\section{Fazit}

Der Paritätische Wohlfahrtsverband hat in der Debatte um die Neuorganisation der Strukturen nach dem SGB II gute Gründe für kommunale Zuständigkeiten geltend gemacht. Dass nach dem aktuellen politischen Diskussionsstand die 69 Optionskommunen fortbestehen können, ist zumindest ein Teilerfolg.

Eine Grundgesetzänderung muss die Zahl der Optionskommunen offen lassen, damit in Zukunft vielleicht über eine weitergehende Kommunalisierung der Hilfen für Arbeitslose neu entscheiden wird. Das ist umso wichtiger, als die vorgesehenen Nachfolgeorganisationen der ARGEN den Optionskommunen vermutlich keinesfalls überlegen sein werden. Vielmehr gibt es zahlreiche Unwägbarkeiten zu beseitigen, damit die strukturellen Voraussetzungen der neuen ARGEN an die Vorteile der Optionskommunen heranreichen werden.

Aber auch die bestehenden Optionskommunen sind gefordert, ihren strukturellen Vorsprung zu nutzen und ihre Förderpraxis zu verbessern. Für einen stärkeren sozialintegrativen Ansatz nach dem SGB II müssen die Kommunen außerdem mehr finanzielle Ressourcen einbringen. Und es bedarf neuer Spielregeln für ein finanzielles Fairplay von Bund und Kommunen in der Umsetzung von Eingliederungsleistungen.

\section{Anmerkungen}

(1) Siehe $\$ 6$ c SGB II.

(2) Siehe auch Heike Förster: Ausbildungschancen junger Menschen im SGB II, Expertise, 2008.

(3) Bundesagentur für Arbeit (Hg.) 2007: SGB II - Sozialgesetzbuch Zweites Buch, Grundsicherung für Arbeitssuchende. Jahresbericht 2007.

(4) Deutscher Landkreistag (Hg.): Leitlinien zur Umsetzung der sozialen Leistungen nach dem SGB II, Juni 2008. 